\title{
Analysis of the Fuel Efficiency of a Hybrid Electric Drive with an Electric Power Splitter
}

\author{
D. Čundev
}

This paper presents the results of an analysis of the fuel efficiency of a hybrid electric car drive, with an electric power splitter based on a double rotor synchronous permanent magnet generator. The results have been obtained through a precisely determined mathematical model and by simulating the characteristics of all essential values for the entire drive. This work is related to the experimental working stand for electric and hybrid car drive research, which has been developed at the Faculty of Electrical Engineering (FEE) at CTU in Prague.

Keywords: Hybrid drive, fuel efficiency, European driving schedule, electric power splitter, electric traction motor, super-capacitor.

\section{Introduction}

Improving the fuel efficiency of passenger vehicles is the ultimate goal of a large scientific and technical community. The main purpose of a hybrid electric drive is to achieve a higher efficiency in energy transmission from internal combustion engine (ICE) to the traction wheels of the vehicle. The hybrid electric vehicle (HEV) is a transient solution from standard internal combustion engine driven cars to all-electric driven vehicles (EV). Some car manufacturing companies already have successful commercial models, but all are based on significantly different technological solution.

In the Josef Bozek Research Center for Engine and Automotive Engineering (RCJB) at CTU in Prague there is an ongoing project to develop a hybrid electric drive. For this purpose, an experimental working stand has been set up at the Department of Electric Drives and Traction at the Faculty for Electrical Engineering (FEE). The main innovative features of this hybrid drive are the use of a super-capacitor (SC) as an accumulation unit, and an electric power splitter (EPS).

Commercial hybrid electric cars, for splitting energy from ICE use a planetary gear and a separate electrical generator for the electrical power supply of the traction motor and for charging the battery. In the hybrid electric system developed at CTU in Prague, power splitting is performed entirely electrically, with the use of EPS. Also, instead of a chemical battery for accumulating the breaking kinetic energy, a super-capacitor is used in this stand as a new technological element for electrical energy storage. This enables energy to be stored without transformation from electrical to chemical and back. This leads to higher efficiency in energy form transformation.

\section{$2 \mathrm{HEV}$ with electric power splitter}

A schematic representation of the working concept of HEV with EPS and a super-capacitor is shown in Fig. 1.

The internal combustion engine is the main and only power source of a vehicle that produces mechanical power Pice. EPS is a special type of synchronous generator with two rotating parts (a classic permanent magnet rotor and a rotating stator). The rotor is firmly coupled to the drive-shaft of ICE, and the stator of the EPS is firmly coupled to the transmission that leads to the car wheels and rotates at a

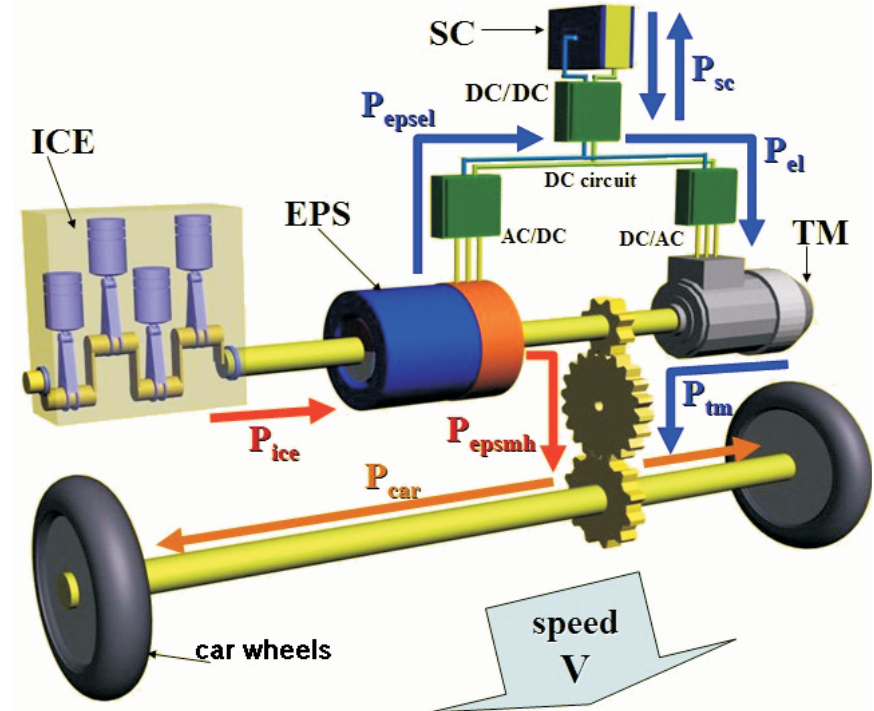

Fig. 1: Schematic representation of the HEV

speed proportional to the velocity of the vehicle (speed V). This technical solution enables the ICE to operate on the optimal revolutions during the entire driving schedule.

Mechanical power $P_{\text {ice }}$ is divided into electrical power $P_{\text {epsel }}$ and mechanical power $P_{\text {epsmh }}$. The induction traction motor (TM) has been inserted on the shaft of the EPS rotating stator, and is the main electric propulsion to the vehicle. EPS and TM are electrically connected through two traction $\mathrm{AC} / \mathrm{DC}$ and DC/AC power converters, with an intermediate DC link. SC is connected to the DC link via a charging and discharging DC-DC converter. TM is powered by $P_{\mathrm{el}}$, which is generated in EPS $\left(P_{\text {epsel }}\right)$ and by additional power from SC $\left(P_{\mathrm{sc}}\right)$ :

$$
P_{\mathrm{el}}=P_{\text {epsel }}+P_{\mathrm{sc}} .
$$

The traction motor TM produces mechanical power $P_{\text {tm }}$ which, with mechanical power $P_{\text {epsmh }}$ added from EPS, is transmitted to the car wheels. The total power $P_{\text {car }}$ propelling the car is expressed by the formula:

$$
P_{\text {car }}=P_{\text {epsmh }}+P_{\mathrm{tm}} \text {. }
$$

When the car is braking, TM changes function from motor to generator. In this way, the decelerating energy of the car 
can be partially converted into electric energy, which is accumulated in SC and can later be used for acceleration in the following driving cycles.

\section{Experimental working stand}

In order to perform laboratory tests on this HEV concept, an experimental working stand was se up in the laboratory. The scheme of this laboratory stand is shown in Fig. 2. The main functional units of the stand are the same as the units of the HEV concept, shown in Fig. 1.

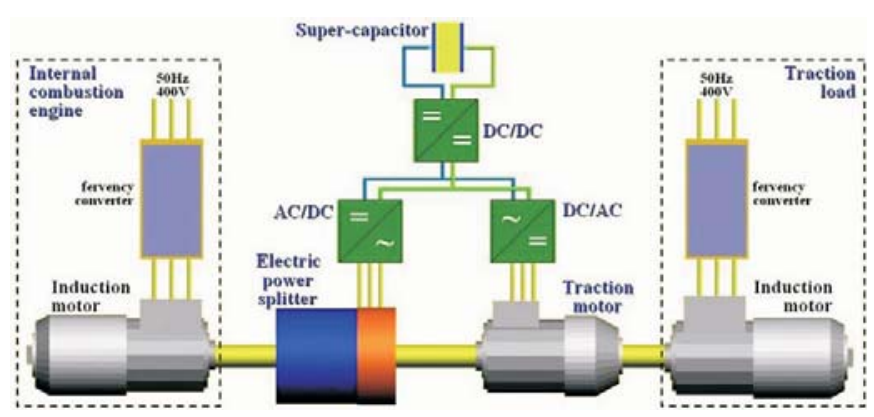

Fig. 2: Experimental working stand for HEV

In this HEV laboratory model, ICE and the car wheels are substituted by two regulated induction motors. The internal combustion engine is simulated by a controlled electric AC induction motor. The traction load is simulated with another controlled AC induction motor.

The entire working stand consists of four electric machines (shown in Fig. 3), five semiconductor power control units, a super-capacitor and all the necessary instrumentation, control, power supply and protection equipment.

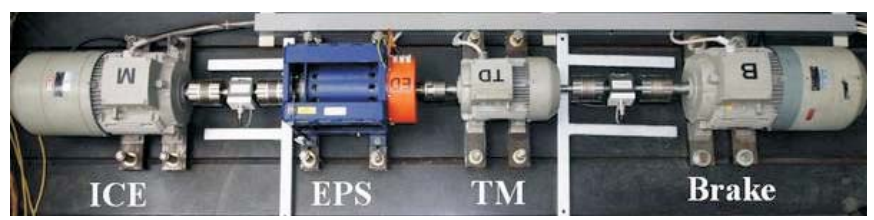

Fig. 3: Electric machines of the laboratory working stand

\section{Vehicle fuel efficiency}

The fuel efficiency of passenger vehicles is defined as the amount of fuel required $\left(L_{\mathrm{car}}[\right.$ liter] $)$ for a driven car trajectory distance $\left(S_{\mathrm{car}}[\mathrm{km}]\right)$. In Europe, this value is measured according to a predetermined working regime defined as the European driving schedule (EDS).

EDS consists of a $1200 \mathrm{sec}$. driving schedule, which combines 800 seconds of urban and 400 seconds of highway driving (Fig. 4).

The urban driving consists of four repeated cycles, each with three different acceleration, idle and deceleration regimes. Highway driving is continuous driving with predetermined changes in speed V. This is the standardized European driving schedule for calculating the consumption and vehicle efficiency.

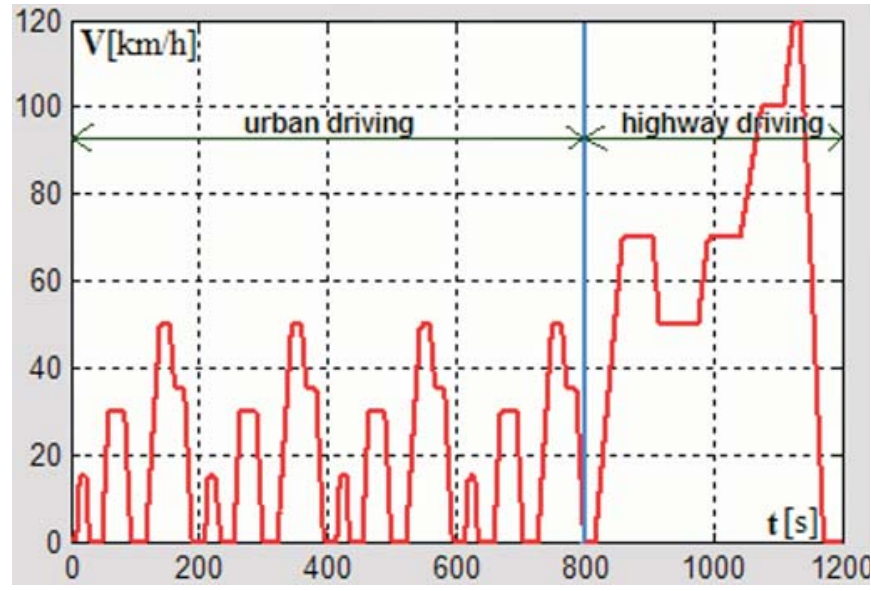

Fig. 4. European driving schedule EDS

The vehicle drives the first 800 sec. (urban driving), and the total driven distance is measured or calculated $\left(\mathrm{S}_{\mathrm{car}}\right)$. This value is divided by the amount of fuel consumed $\left(\mathrm{L}_{\text {car }}\right)$ and the consumption $Q_{\text {urb }}$ of the vehicle for urban driving is calculated:

$$
Q_{\text {car }}=\frac{S_{\text {car }}}{L_{\text {car }}}
$$

The same approach is used for calculating the consumption $Q_{\mathrm{hgw}}$ for highway driving (EDS cycle from 800 to $1200 \mathrm{sec}$.). To calculate the combined consumption $Q_{\text {com }}$ (urban and highway) for the entire EDS (1200 sec.), car trajectory $S_{\text {car }}$ and fuel consumed $L_{\text {car }}$ are calculated.

\section{Simulation of the EDS}

A simulation has been made by means of the Matlab programming interface. The main approach in this simulation is to determine the energy fluctuations in the hybrid drive during the driving schedule. For this purpose, the function and behavior of each component of the system is determined and taken into account, e.g., aerodynamic resistance, rolling resistance between the tires and the road surface, density of the ambient air, cross-sectional area of the vehicle, coefficient of drag, etc.

The kinematic model has been mathematically created for the predetermined car specifications, e.g., car weight, efficiency of transmission, ICE fuel consumption and output power, number of accumulative units - SC, and the efficiency of the electric power converters. For example, for each time unit, $\left(\Delta t_{n}=10^{-3}[\mathrm{sec}]\right)$ of the drive it has been calculated the acceleration $a_{n}$, car trajectory distance $S_{n}$, needed acceleration force $F_{a}$, and corresponding energy $W_{n}$. By means of these calculated values, acceleration power $P_{a}$ can be calculated for each subinterval $\Delta t_{n}$. The aerodynamic and rolling resistance between the tires and the road surface has been compensated by calculating the additional power $P_{v}$, which depends on speed $V(t)$. The total power that a hybrid vehicle needs to provide is the sum of acceleration power $P_{a}$ and speed power $P_{v}$ :

$$
P_{\text {car }}=P_{a}+P_{v}
$$

The program calculates all these values according to EDS. The data is presented in characteristics which are functions of time $t$. The power on the drive-shaft of the internal combus- 
tion engine $P_{\text {ice, }}$, follows the required driving power $P_{\text {car }}$, which is shown in Fig. 5. This is the main advantage of a hybrid electric drive that can not be performed in normal cars, where the ICE must instantly and rapidly change the working regime according to the actual power demand. This leads to high fuel consumption and low efficiency. In HEVs, ICE works only when the working regime demands significant power, which is not the case with conventional cars, where ICE works all the time, even when the car is not moving, e.g., in normal urban driving.

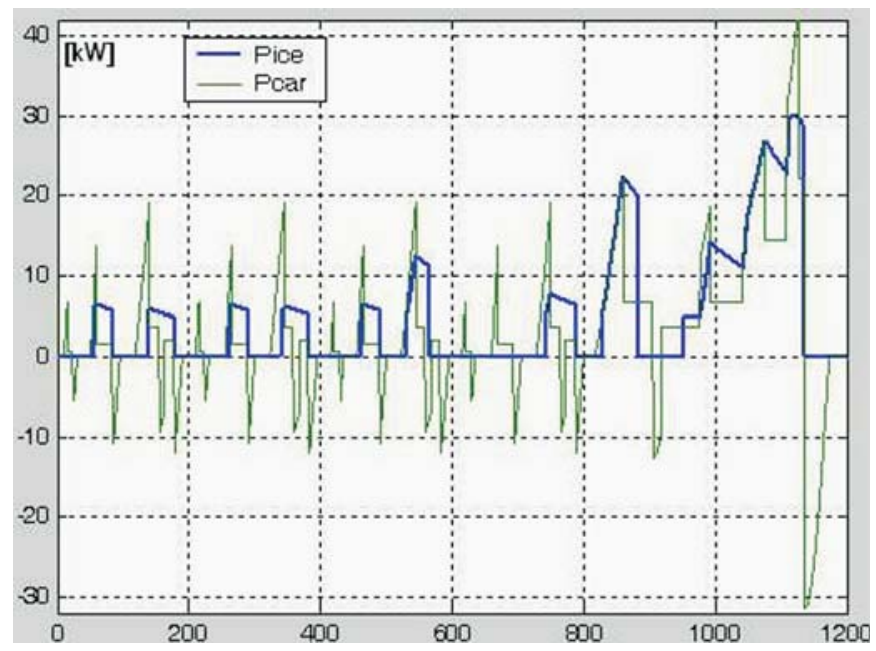

Fig. 5: $P_{\text {car }}$ and power produced on the drive shaft of the ICE

ICE provides power for driving the vehicle and also for charging SC and keeping $U_{\mathrm{sc}}$ above the critical minimal level $U_{\text {scmin }}$. Therefore $P_{\text {ice }}$ depends on the actual energy volume $W_{\mathrm{sc}}$ accumulated in the super-capacitor, which is determined from the actual SC voltage $U_{\mathrm{sc}}$ :

$$
W_{\mathrm{sc}}=\frac{C \cdot U_{\mathrm{sC}}^{2}}{2}
$$

\section{Simulation results}

The simulation results are shown in graphs representing the calculated values as a function of time $t$ during the entire driving regime of EDS. Fig. 5 shows the output power $P_{\text {ice }}$ of an internal combustion engine. Fig. 6 presents the actual voltage of SC. According to equation (5), by measuring $U_{\mathrm{sc}}$, the precise data on the accumulated energy in the super-capacitor can be obtained during the entire working regime.

The working regime is determined in such a way that $U_{\mathrm{sc}}$ is maintained between the two critical values $U_{\text {scmin }}$ and $U_{\text {scmax }}$. This is important, because SC must not be overcharged $\left(U_{\mathrm{sc}} \leq U_{\mathrm{scmax}}\right)$ and it must be keep over minimal level $\left(U_{\mathrm{sc}}>U_{\text {scmin }}\right)$ to have an energy reserve.

Low voltage of SC is undesirable, because the current level $\left(I_{\mathrm{sc}}\right)$ of SC is increased and with that the efficiency of SC rapidly decreases. The efficiency (Fig. 8) of the recuperative circuits of HEV (power control units DC-DC and SC) is proportional to the current flow in those circuits.

When simulating a of power drive system, is essential to calculate precisely the efficiency of each component where there is energy transformation. We have calculated the effi-

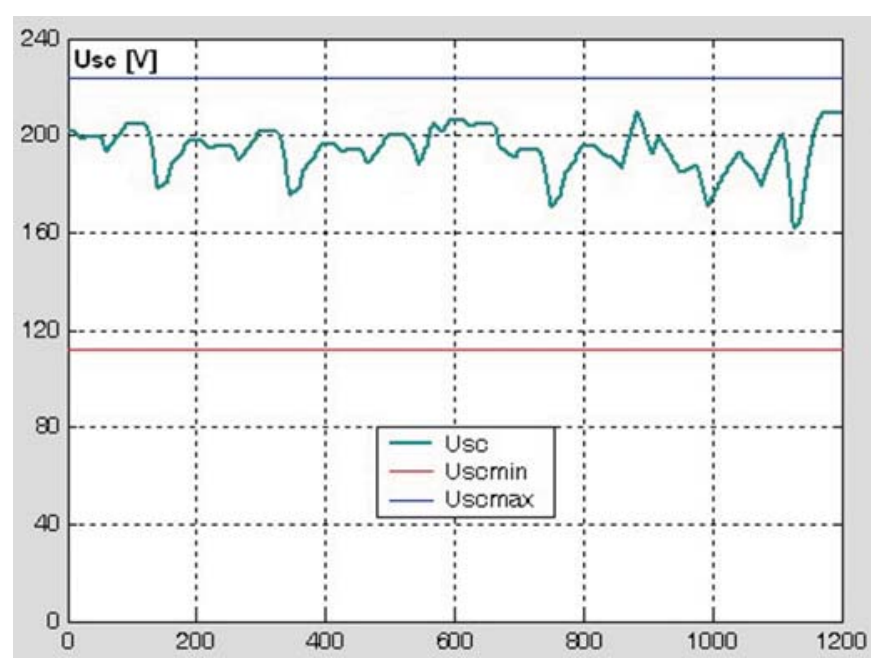

Fig. 6: Voltage $U_{\mathrm{sc}}$ in function of time $t$

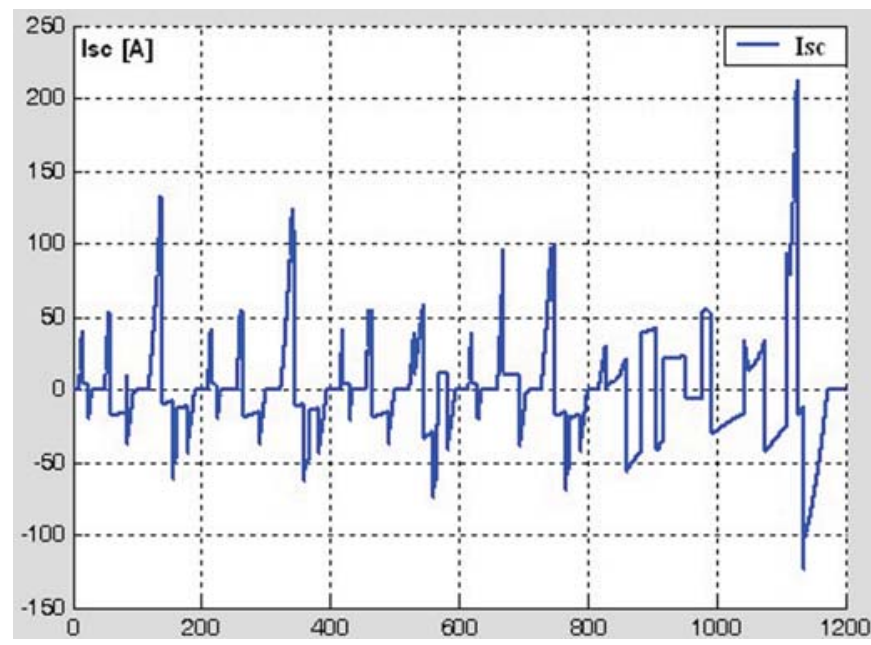

Fig. 7: Current $I_{\mathrm{sc}}$ as a function of time $t$

ciencies of ICE (fuel consumption according to the power demand $P_{\text {ice }}$ and the revolutions of the drive-shaft), the efficiency of EPS (the electrical $P_{\text {epsel }}$ and mechanical $P_{\text {epsmh }}$ power transformation), the efficiency of TM and the efficiency of transmission and SC (Fig. 8).

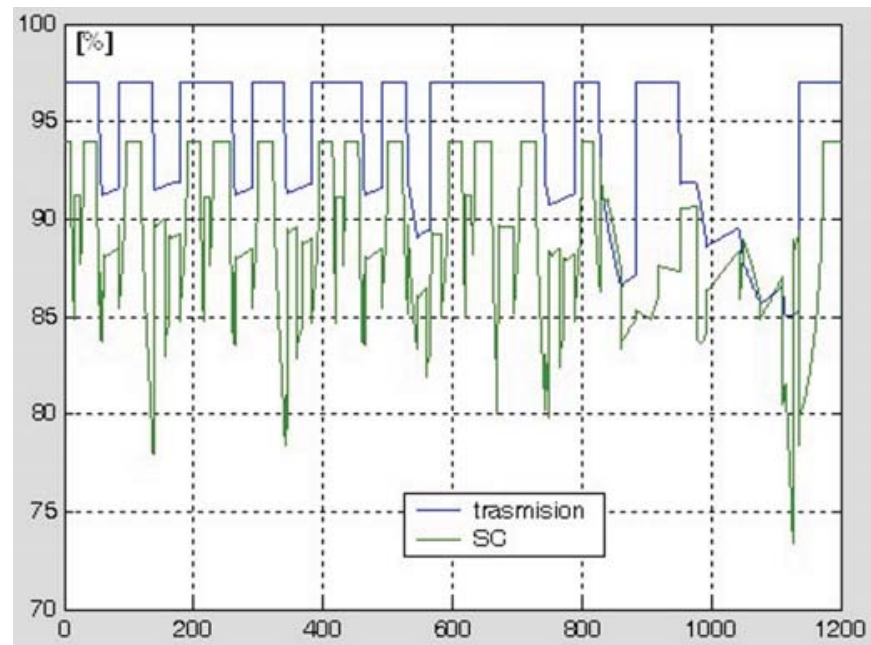

Fig. 8: Efficiency of transmission and SC 
Fuel consumption is measured in each time interval. Knowing the total fuel consumed in the end drive regime (in EDS $t=800[\mathrm{~s}$ ] for the urban regime and $t=1200[\mathrm{~s}]$ for the combined drive regime) and the total distance driven, we can calculate the consumption of the hybrid-electric drive is calculated. For urban driving consumption is 25.6 [km/l], for the highway $21.4[\mathrm{~km} / \mathrm{l}]$. For combined driving it is $23.2[\mathrm{~km} / \mathrm{l}]$. Table 1 compares the values those for standard drive cars with similar characteristics.

Table 1: Fuel consumption

\begin{tabular}{|l|c|c|c|}
\hline \multicolumn{1}{|c|}{$[\mathrm{km} / \mathrm{l}]$} & urban & highway & combined \\
\hline Hybrid-electric vehicle & 25.6 & 21.4 & 23.2 \\
\hline Standard ICE vehicle & 13.7 & 19.2 & 17.2 \\
\hline
\end{tabular}

\section{Conclusions}

The results show the main specifications of this new concept of hybrid electric drive according to the European driving schedule. This mathematical concept and these simulations provide the analytical results which prove the eligibility of this new technological solution. The results will also be used for tests on the real HEV working stand, which is constructed at FEE, Czech Technical University in Prague.

The results present the operating conditions of all systems during the driving regime. The changes in the values as a function of time give an overview of the energy fluctuation in each component of the system.

The final numerical results for fuel consumption show the significance of this new technological approach. Enabling vehicles to drive a greater distance using the same amount of fuel is the main task in increasing the efficiency of passenger cars.

\section{Acknowledgement}

The research described in this paper was supervised by doc. Ing. Pavel Mindl, CSc, Department of Electrical Drives and Traction, FEE CTU in Prague. It was supported by Josef Božek Center of Engine and Automotive Engineering. The project has been supported by the Czech Ministry of Education grant.

\section{References}

[1] Čundev, D., Mindl, P.: Driving Characteristics for Hybrid Electric Drive with Super-capacitor as Energy Storage Unit. Progress in Electromagnetics Research Symposium PIERS2007, Prague, 2007.

[2] Čeřovský, Z., Mindl, P.: Hybrid Drive with Super-capacitor Energy Storage, FISITA Conference, Barcelona, F193m, 2004.

[3] Čeřovský, Z., Mindl, P., Flígl, V, Halámka, Z., Hanuš, P., Pavelka, V.: Power Electronics in Automotive Hybrid Drives, EPE-PEMC2002, Dubrovnik, Croatia Conference, September 2002.

[4] Čeřovský, Z., Mindl, P.: Double Rotor Synchronous Generator Used as Power Splitting Device in Hybrid Vehicles, $31^{\text {st }}$ FISITA World Automotive Congress, Yokohama 2006.

Ing. Dobri Čundev

e-mail: cunded1@fel.cvut.cz

Department of Electric Drives and Traction

Czech Technical University in Prague

Faculty of Electrical Engineering

Technická 2

16627 Prague, Czech Republic 